Supporting information

for

\title{
A composite hybrid quasi-solid electrolyte for high-energy lithium metal
}

\section{batteries}

Yanfang Zhai ${ }^{a}$, Guanming Yang ${ }^{a}$, Zhong Zeng ${ }^{a}$, Shufeng Song ${ }^{a,}{ }^{*}$, Shuai Li $^{b}$, Ning $H u^{c, *}$, Weiping Tang ${ }^{d}$, Zhaoyin Wen ${ }^{e}$, Li Lu ${ }^{f}$, Janina Molenda ${ }^{g}$

${ }^{a}$ College of Aerospace Engineering, Chongqing University, Chongqing 400044,

China

${ }^{\mathrm{b}}$ Guangdong Provincial Key Laboratory of Energy Materials for Electric Power,

Southern University of Science and Technology, Shenzhen 518055, Guangdong,

China

c State Key Laboratory of Reliability and Intelligence Electrical Equipment, National Engineering Research Center for Technological Innovation Method and Tool, and School of Mechanical Engineering, Hebei University of Technology, Tianjin 300401,

China

d State Key Laboratory of Space Power-Sources Technology, Shanghai Institute of Space Power-Sources, Shanghai 200245, China

e CAS Key Laboratory of Materials for Energy Conversion, Shanghai Institute of Ceramics, Chinese Academy of Sciences, Shanghai 200050, China

${ }_{\mathrm{f}}^{\mathrm{f}}$ Department of Mechanical Engineering, National University of Singapore, 117575, Singapore

${ }^{\mathrm{g}}$ AGH Univ Sci \& Technol, Fac Energy \& Fuels, Al Mickiewicza 30, PL-30059 
Krakow, Poland

*Corresponding author.

E-mail address: sfsong@cqu.edu.cn (S. Song); ninghu@cqu.edu.cn (N. Hu) 


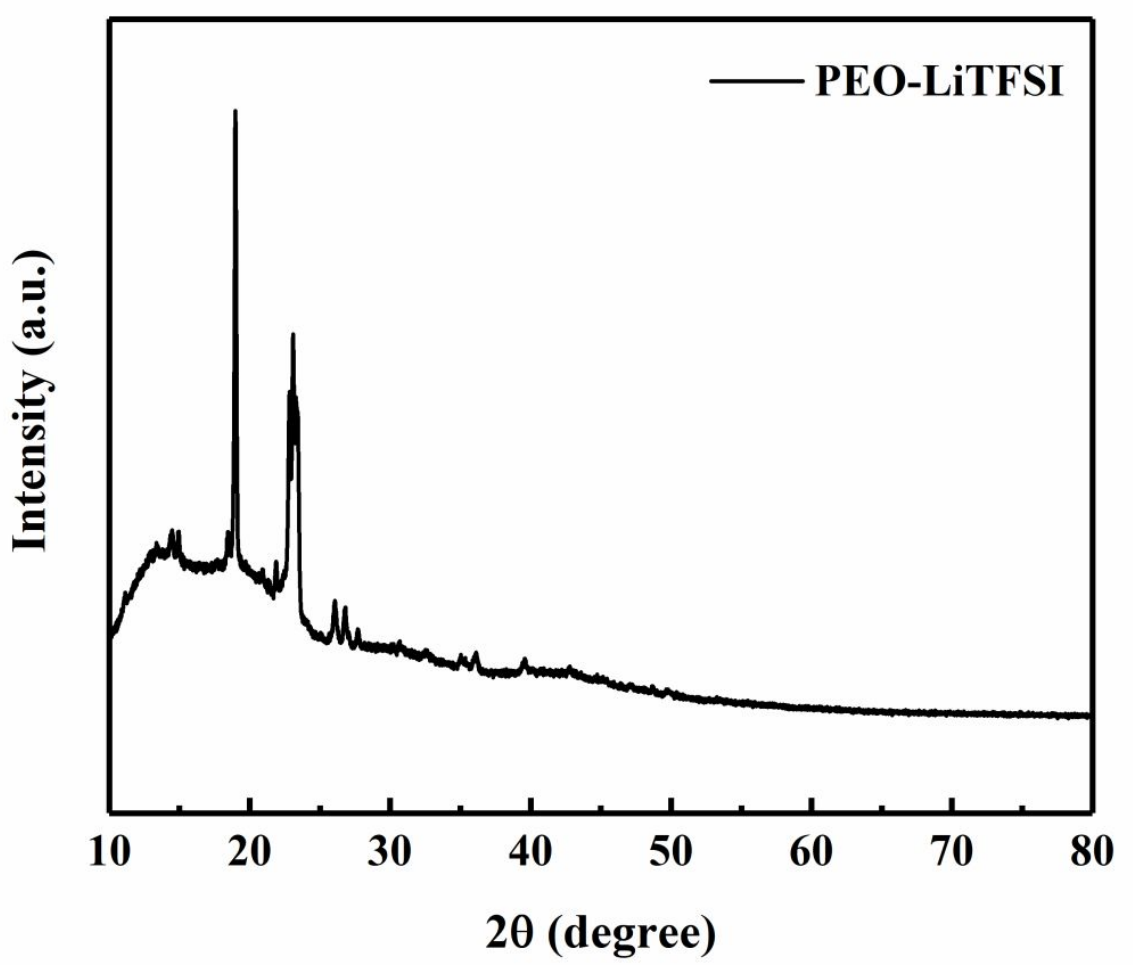

Figure S1. The XRD profile of PEO-LiTFS 

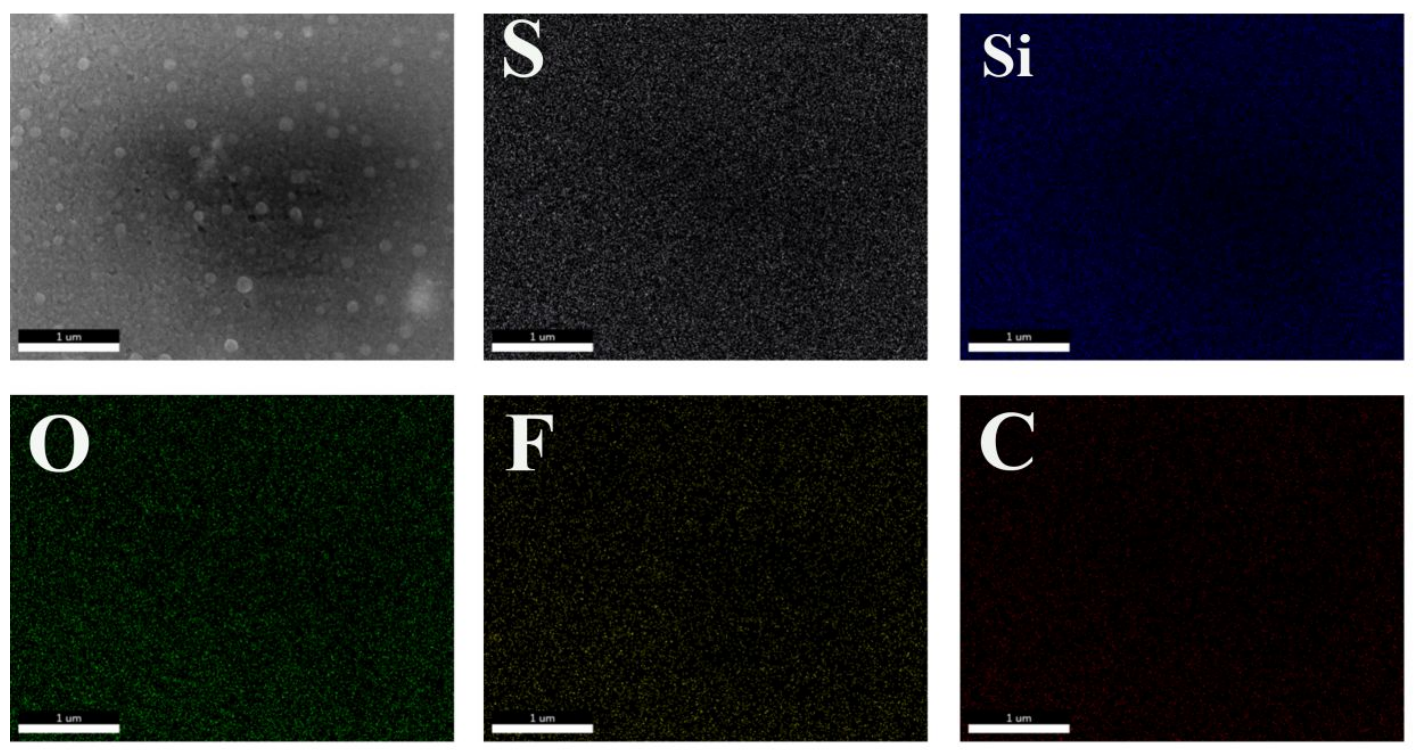

Figure S2. The EDS of the composite hybrid quasi-solid electrolyte. The scale bar is $1 \mathrm{um}$.

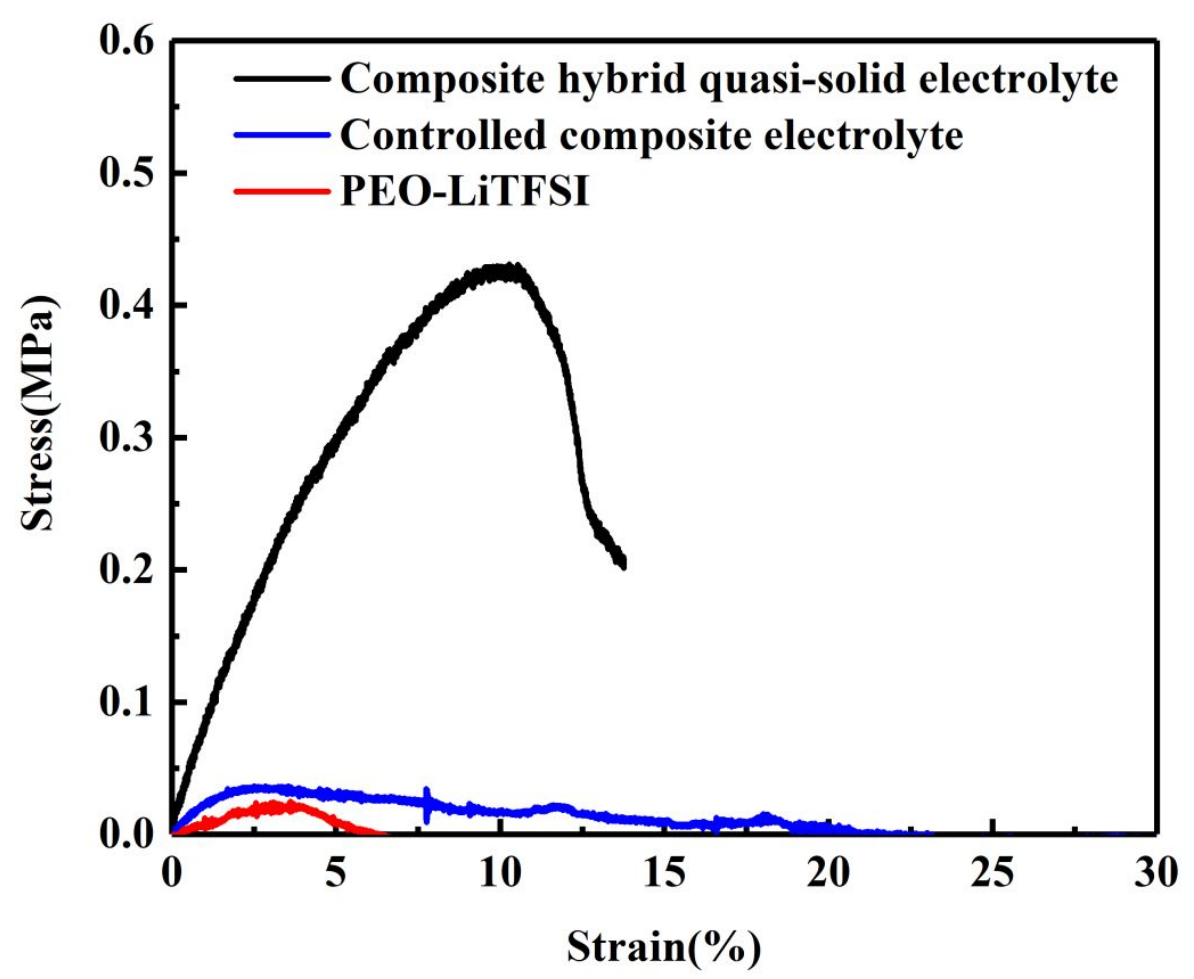


Figure S3 Stress-strain curves of composite hybrid quasi-solid electrolyte, controlled composite electrolyte and PEO-LiTFSI

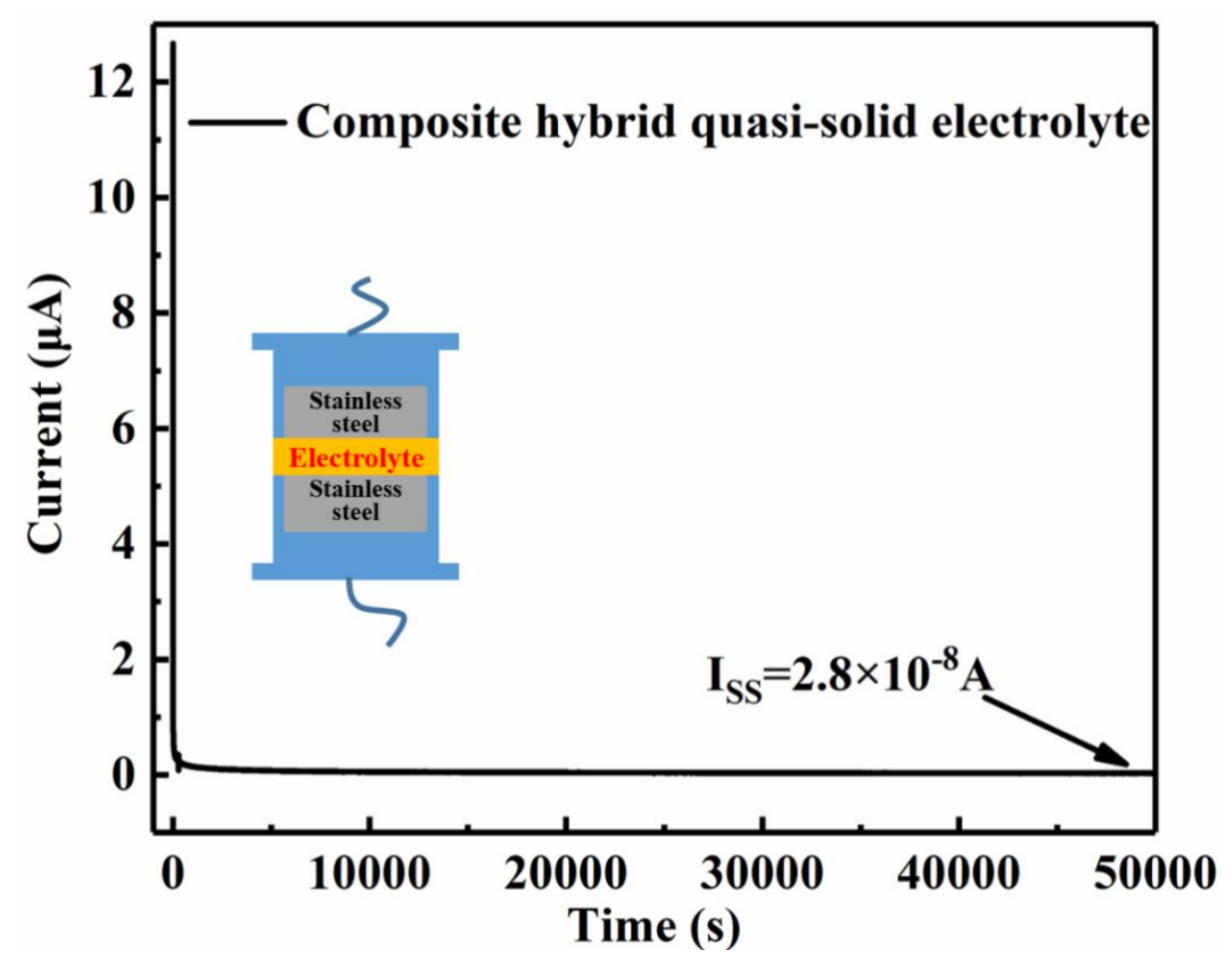

Figure S4. Polarization current-time curve in a SS/electrolyte/SS cell. 